\title{
Synthesis and biological Evaluation of Some Abbreviated New pyrimidine derivatives
}

\author{
Jatin Vora ${ }^{1}$, Kartik Vyas ${ }^{2}$
}

${ }^{1}$ Department of Chemistry, Sheth L. H. Science College, Mansa, India (Scholars of Gujarat University, Ahmedabad), Gujarat, India

${ }^{2}$ Department of Chemistry, Sheth L. H. Science College, Mansa, Gujarat, India

\section{ABSTRACT}

A cogent synthesis of completely new compound series of by 6-(3,5-bis(trifluoromethyl)phenyl)-4-(4chlorophenyl)-3,4-dihydro pyrimidine-2(1H)-one (Biginelli reaction) was achieved by continue heating of (E)-1-(3,5-bis(trifluoromethyl)phenyl)-3-(substituted phenyl)prop-2-en-1-one and urea for 5 hours with 40\% $\mathrm{KOH}$ and ethyl alcohol. (E)-1-(3,5-bis(trifluoromethyl)phenyl)-3-(substituted phenyl)prop-2-en-1-one is produced by Claisen-Schmidt Condensation. In this condensation 1-(3,5-bis(trifluoromethyl)phenyl)ethanone and different aldehyde are mixed. All the novel compound series were characterized by infrared and $1 \mathrm{H}$ nuclear magnetic resonance and mass spectroscopic techniques and by elemental analyses. The newly synthesized compounds were evaluated for their antibacterial and antifungal activity

Keywords : (E)-1-(3,5-bis(trifluoromethyl)phenyl)-3-(substituted phenyl)prop-2-en-1-one, Urea, KOH and Ethanol.

\section{INTRODUCTION}

The biginelli rection is the most efficient reaction for the preparation of di hydropyrimidines. Which is the multi-component and acid-catalyzed reaction [1]. The acid which is used in this reaction is copper (II) trifluoroacetate hydrate [2]. And boron trifluoride. The product forming during this method is used as a calcium channel blocker [3]. Antihypertensive agents antimicrobial and antifungal agents. In 1987, Atwal et al.[4][5] reported a modification to the Biginelli reaction that consistently generated higher yields. Atul Kumar has reported first enzymatic synthesis for Biginelli reaction via yeast catalyzed protocol in high yields.[6] The key advantage of the present method is the capability to allow variability of functional groups, short reaction times, easy workup, high yields, thus providing economic and environmental advantages. [7] 4 - (benzyloxy) - 3 - methoxy benzaldehyde, $\mathrm{N}$ - (substituted phenyl) - 3 oxobutanamide and 2H-1,2,4-triazole-3-amine are three-component which are mainly responsible for highly efficient condensation

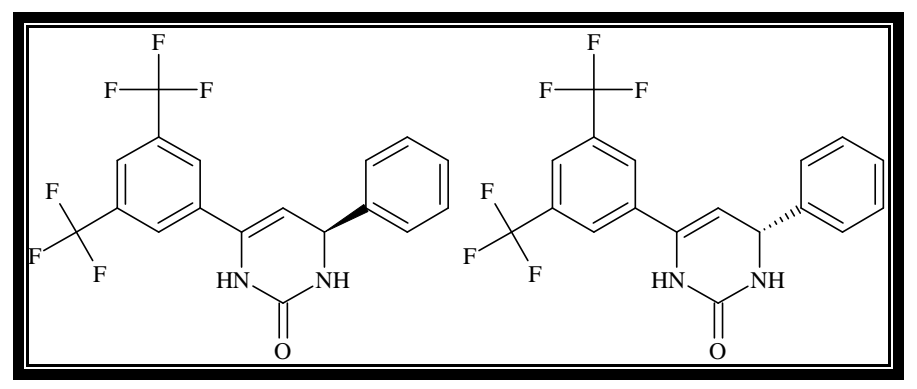




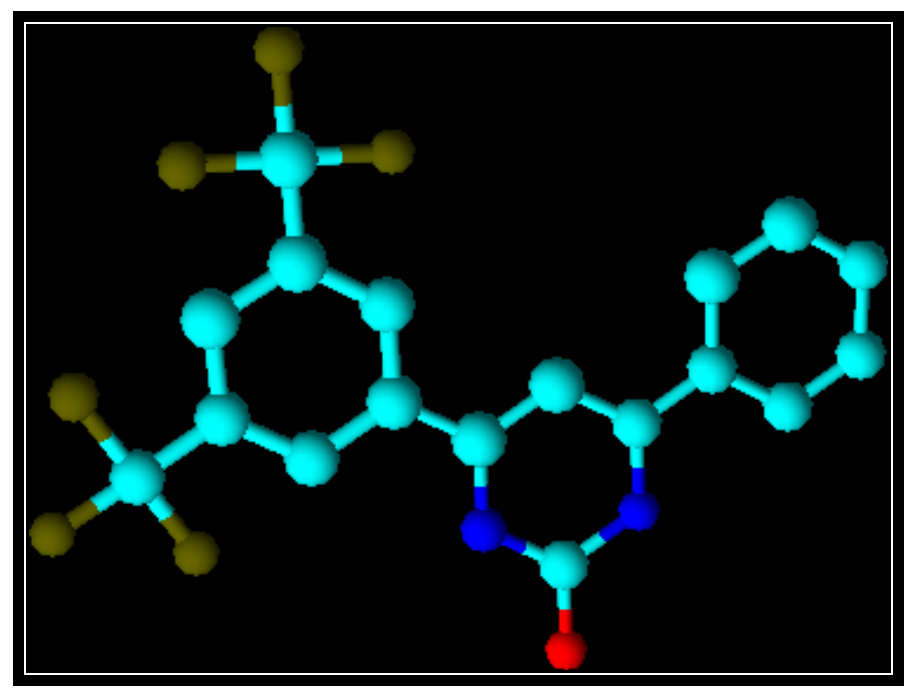

Figure 1.3D Structure

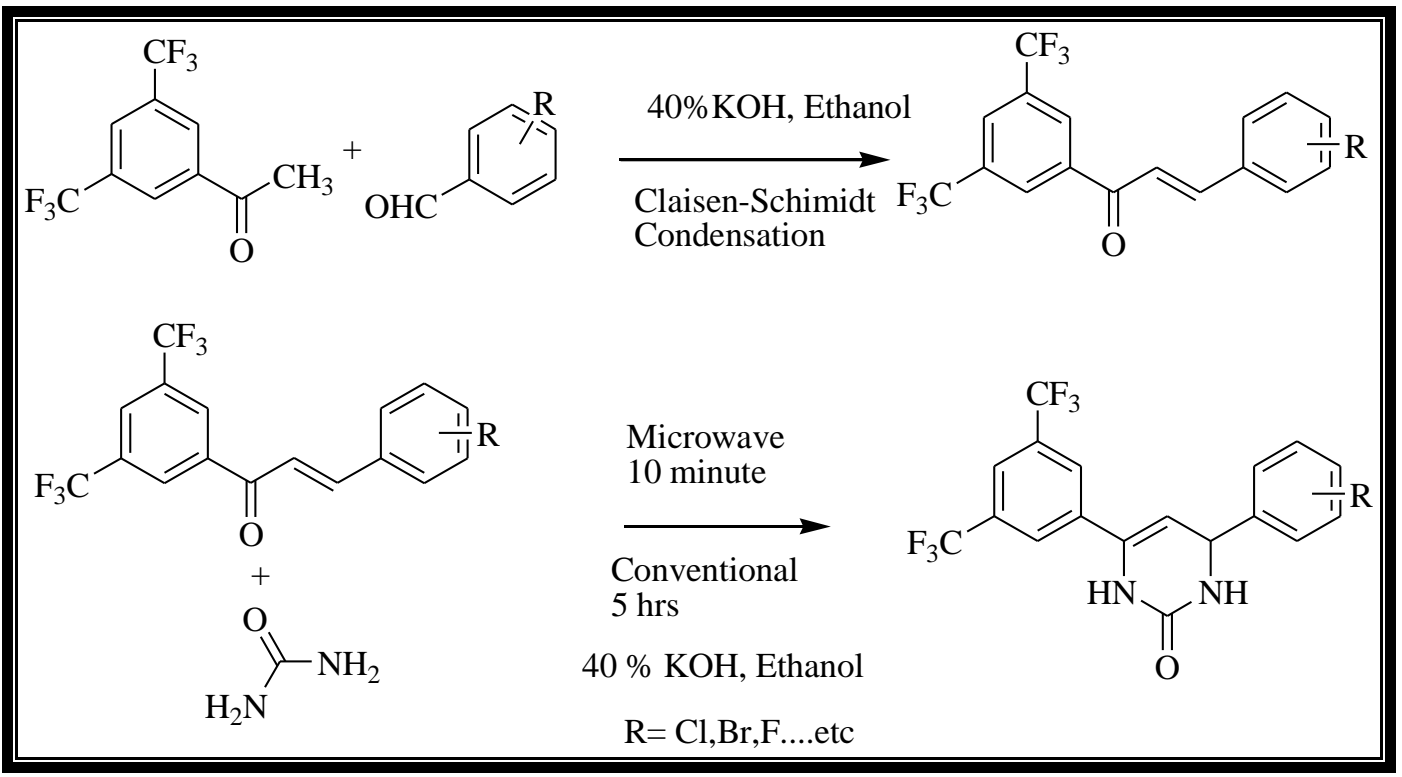

\section{EXPERIMENTAL}

\section{Typical untried procedure}

A mixture of (E)-1-(3,5-bis(trifluoromethyl)phenyl)3-(substituted phenyl)prop-2-en-1-one and Urea was refluxed in $40 \% \mathrm{KOH}$ in ethanol $(20 \mathrm{ml})$ on water bath for 5 hours. The solvent was distilled out under vacuum and neutralized with $20 \% \mathrm{HCl}$, filter out the solid product and crystalline from ethanol.

\section{6-(3,5-bis(trifluoromethyl)phenyl)-4-(4-} chlorophenyl)-3,4-dihydropyrimidin-2(1H)-one (4a) M.P. 160두 Yield: 70\%; IR $(\mathrm{KBr})\left(\mathrm{cm}^{-1}\right): 3092(\mathrm{~N}-\mathrm{H})$, 2916 (C-H), 1610 (C=O), 1588, 1462 (C=C), 1095 (CF); $754(\mathrm{C}-\mathrm{Cl}) ;{ }^{1} \mathrm{H}$ NMR (DMSO- $\left.d 6\right) \delta$ ppm: $\delta 5.50(\mathrm{~s}$, $2 \mathrm{H}$, - $\mathrm{CH}$ - of pyrimidine ring), 6.96-6.98 $(\mathrm{m}, 1 \mathrm{H}, \mathrm{Ar}-$ $\mathrm{H})$, 7.23-7.33 (m, 2H, Ar-H), 7.43-7.44 (s, 1H, -Ar$\mathrm{H}), 7.53-7.57$ (s, 2H, $-\mathrm{Ar}-\mathrm{H}), 8.31-8.33$ (s, 2H, $-\mathrm{NH}$ of pyrimidine), 8.61 (s, $1 \mathrm{H},-\mathrm{Ar}-\mathrm{H}$ ); MS: $\mathrm{m} / z$ 420. Anal. found: C, 51.79; $\mathrm{H}, 2.48 ; \mathrm{Cl}, 8.87 ; \mathrm{F}, 27.14 ; \mathrm{N}, 6.53$. $\mathrm{C}_{18} \mathrm{H}_{11} \mathrm{ClF}_{6} \mathrm{~N}_{2} \mathrm{O}$ requires: $\mathrm{C}, 51.38 ; \mathrm{H}, 2.64 ; \mathrm{Cl}, 8.43 ; \mathrm{F}$, 27.09; N, $6.66 \%$. 
6-(3,5-bis(trifluoromethyl)phenyl)-4-(4-

fluorophenyl)-3,4-dihydropyrimidin-2(1H)-one (4b)

M.P. 169ㄷ; Yield: 74\%; IR(KBr)(cm-1): $3086(\mathrm{~N}-\mathrm{H})$, $2931(\mathrm{C}-\mathrm{H}), 1641(\mathrm{C}=\mathrm{O})$, 1581, $1465(\mathrm{C}=\mathrm{C}), 1074(\mathrm{C}-$ F); 727 (C-Cl); ${ }^{1} \mathrm{H}$ NMR (DMSO-d6) $\delta$ ppm: $\delta 5.49$ (s, $2 \mathrm{H}$, - $\mathrm{CH}$ - of pyrimidine ring), 7.35-7.40 (m, $4 \mathrm{H}, \mathrm{Ar}-$ $\mathrm{H})$, 7.62-7.67 (m, 1H, Ar-H), 7.80 (s, 2H, -Ar-H), 7.93-7.95 (s, 2H, -NH of of pyrimidine); MS: $\mathrm{m} / z 404$. Anal. found: C, 53.40; H, 2.84; F, 32.15; N, 6.63. $\mathrm{C}_{18} \mathrm{H}_{11} \mathrm{~F}_{7} \mathrm{~N}_{2} \mathrm{O}$ requires: $\mathrm{C}, 53.48 ; \mathrm{H}, 2.74 ; \mathrm{F}, 32.90 ; \mathrm{N}$, $6.93 \%$.

\section{6-(3,5-bis(trifluoromethyl)phenyl)-4-(4-}

\section{bromophenyl)-3,4-dihydropyrimidin-2(1H)-one (4c)}

M.P. 166우 Yield: 70\%; IR(KBr)(cm-1): $3089(\mathrm{~N}-\mathrm{H})$, $2964(\mathrm{C}-\mathrm{H}), 1668$ (C=O), 1577, 1460 (C=C), 1069 (CF); 733 (C-Cl); ${ }^{1} \mathrm{H}$ NMR (DMSO-d6) $\delta$ ppm: $\delta 5.43$ (s, $2 \mathrm{H}$, - $\mathrm{CH}$ - of pyrimidine ring), 7.33-7.38 (m, $4 \mathrm{H}, \mathrm{Ar}-$ $\mathrm{H})$, 7.62-7.65 (m, 1H, Ar-H), 7.77 (s, 2H, -Ar-H), 7.88-7.90 (s, 2H, -NH of of pyrimidine); MS: $m / z 465$. Anal. found: C, 46.49; H, 2.39; Br, 17.11; F, 24.51; N, 6.07. $\mathrm{C}_{18} \mathrm{H}_{11} \mathrm{BrF}_{6} \mathrm{~N}_{2} \mathrm{O}$ requires: $\mathrm{C}, 46.47 ; \mathrm{H}, 2.38 ; \mathrm{Br}$, 17.18; F, 24.50; N, 6.02\%.

\section{6-(3,5-bis(trifluoromethyl)phenyl)-4-(3-}

chlorophenyl)-3,4-dihydropyrimidin-2(1H)-one (4d)

M.P. 162우 Yield: 63\%; $\mathrm{IR}(\mathrm{KBr})\left(\mathrm{cm}^{-1}\right): 3092(\mathrm{~N}-\mathrm{H})$, $2916(\mathrm{C}-\mathrm{H}), 1610$ (C=O), 1588, 1462 (C=C), 1095 (CF); 754 (C-Cl); ${ }^{1} \mathrm{H}$ NMR (DMSO- $\left.d 6\right) \delta$ ppm: $\delta 5.50$ (s, $2 \mathrm{H}$, - $\mathrm{CH}$ - of pyrimidine ring), 6.87-6.90 (m, $1 \mathrm{H}, \mathrm{Ar}-$ $\mathrm{H})$, 7.20-7.25 (m, 2H, Ar-H), 7.43-7.44 (s, 1H, -Ar$\mathrm{H}), 7.50-7.51$ (s, 2H, -Ar-H), 8.22-8.22 (s, 2H, -NH of pyrimidine), 8.71 (s, $1 \mathrm{H},-\mathrm{Ar}-\mathrm{H})$; MS: $\mathrm{m} / z$ 420. Anal. found: C C, 51.34; H, 2.60; Cl, 8.41; F, 27.11; N, 6.42. $\mathrm{C}_{18} \mathrm{H}_{11} \mathrm{ClF}_{6} \mathrm{~N}_{2} \mathrm{O}$ requires: $\mathrm{C}, 51.38 ; \mathrm{H}, 2.64 ; \mathrm{Cl}, 8.43 ; \mathrm{F}$, $27.09 ; \mathrm{N}, 6.66 \%$.

\section{6-(3,5-bis(trifluoromethyl)phenyl)-4-(3-}

fluorophenyl)-3,4-dihydropyrimidin-2(1H)-one (4e)

M.P. $160^{\circ} \mathrm{C}$; Yield: $66 \%$; $\operatorname{IR}(\mathrm{KBr})\left(\mathrm{cm}^{-1}\right)$ : $3108(\mathrm{~N}-\mathrm{H})$, 2945 (C-H), 1679 (C=O), 1574, 1468 (C=C), 1088 (CF); 741 (C-Cl); ${ }^{1} \mathrm{H}$ NMR (DMSO-d6) $\delta$ ppm: $\delta 5.43$ (s, $2 \mathrm{H}$, - $\mathrm{CH}-$ of pyrimidine ring), 6.93-6.95 (m, $1 \mathrm{H}, \mathrm{Ar}-$ $\mathrm{H})$, 7.29-7.31 (m, 2H, Ar-H), 7.38-7.40 (s, 1H, -Ar$\mathrm{H}), 7.48-7.50$ (s, 2H, -Ar-H), 8.37-8.39 (s, 2H, -NH of pyrimidine), 8.74 (s, $1 \mathrm{H},-\mathrm{Ar}-\mathrm{H})$; MS: $\mathrm{m} / \mathrm{z} 404$. Anal. found: C, 53.47; H, 2.77; F, 32.96; N, 6.89 . $\mathrm{C}_{18} \mathrm{H}_{11} \mathrm{~F}_{7} \mathrm{~N}_{2} \mathrm{O}$ requires: $\mathrm{C}, 53.48 ; \mathrm{H}, 2.74 ; \mathrm{F}, 32.90 ; \mathrm{N}$, $6.93 \%$.

\section{6-(3,5-bis(trifluoromethyl)phenyl)-4-(3-}

bromophenyl)-3,4-dihydropyrimidin-2(1H)-one (4f)

M.P. 170두 Yield: 70\% IR $(\mathrm{KBr})\left(\mathrm{cm}^{-1}\right): 3111(\mathrm{~N}-\mathrm{H})$, 2947 (C-H), 1644 (C=O), 1584, 1465 (C=C), 1090 (CF); 742 (C-Cl); ${ }^{1} \mathrm{H}$ NMR (DMSO-d6) $\delta$ ppm: $\delta 5.59$ (s, $2 \mathrm{H}$, - $\mathrm{CH}$ - of pyrimidine ring), 7.07-7.09 (m, $1 \mathrm{H}, \mathrm{Ar}-$ $\mathrm{H})$, 7.27-7.29 (m, 2H, Ar-H), 7.44-7.46 (s, 1H, -Ar-H), 7.52-7.54 (s, 2H, -Ar-H), 8.43-8.45 (s, 2H, $-\mathrm{NH}$ of pyrimidine), 8.77 (s, $1 \mathrm{H},-\mathrm{Ar}-\mathrm{H})$; MS: $\mathrm{m} / \mathrm{z}$ 465. Anal. found: C, 46.38; H, 2.40; Br, 17.28; F, 24.33; N, 6.11. $\mathrm{C}_{18} \mathrm{H}_{11} \mathrm{BrF}_{6} \mathrm{~N}_{2} \mathrm{O}$ requires: $\mathrm{C}, 46.47 ; \mathrm{H}, 2.38 ; \mathrm{Br}, 17.18 ; \mathrm{F}$, $24.50 ; \mathrm{N}, 6.02 \%$.

\section{6-(3,5-bis(trifluoromethyl)phenyl)-4-(4-}

methylphenyl)-3,4-dihydropyrimidin-2(1H)-one (4g)

M.P. 159 $\mathrm{C}$; Yield: $60 \%$; $\mathrm{IR}(\mathrm{KBr})\left(\mathrm{cm}^{-1}\right): 3123(\mathrm{~N}-\mathrm{H})$, 2967 (C-H), 1658 (C=O), 1568, 1456 (C=C), 1054 (CF); 743 (C-Cl); ${ }^{1} \mathrm{H}$ NMR (DMSO-d6) $\delta$ ppm: $\delta 5.41$ (s, $2 \mathrm{H}$, - $\mathrm{CH}$ - of pyrimidine ring), 6.89-6.91 (m, $1 \mathrm{H}, \mathrm{Ar}-$ $\mathrm{H})$, 7.28-7.30 (m, 2H, Ar-H), 7.32-7.34 (s, 1H, -Ar$\mathrm{H}), 7.43-7.45$ (s, 2H, -Ar-H), 8.42-8.45 (s, 2H, -NH of pyrimidine), 8.88 (s, $1 \mathrm{H},-\mathrm{Ar}-\mathrm{H})$; MS: $\mathrm{m} / \mathrm{z} 400$. Anal. found: C, 57.21; H, 3.64; F, 28.31; N, 7.12. $\mathrm{C}_{19} \mathrm{H}_{14} \mathrm{~F}_{6} \mathrm{~N}_{2} \mathrm{O}$ requires: $\mathrm{C}, 57.01 ; \mathrm{H}, 3.52 ; \mathrm{F}, 28.47 ; \mathrm{N}$, $7.00 \%$. 
6-(3,5-bis(trifluoromethyl)phenyl)-4-(4-

\section{methoxyphenyl)-3,4-dihydropyrimidin-2(1H)-one}

(4h)

M.P. $170^{\circ} \mathrm{C}$; Yield: $52 \%$; $\mathrm{IR}(\mathrm{KBr})\left(\mathrm{cm}^{-1}\right)$ : $3084(\mathrm{~N}-\mathrm{H})$, $2933(\mathrm{C}-\mathrm{H}), 1668(\mathrm{C}=\mathrm{O}), 1579,1446(\mathrm{C}=\mathrm{C}), 1065(\mathrm{C}-$ F); 745 (C-Cl); ${ }^{1} \mathrm{H}$ NMR (DMSO- $d \sigma$ ) $\delta$ ppm: $\delta 5.48$ (s, $2 \mathrm{H}$, - $\mathrm{CH}$ - of pyrimidine ring), 6.88-6.90 $(\mathrm{m}, 1 \mathrm{H}, \mathrm{Ar}-$ $\mathrm{H})$, 7.28-7.30 (m, 2H, Ar-H), 7.40-7.42 (s, 1H, -Ar-H), 7.66-7.68 (s, 2H, -Ar-H), 8.10-8.12 (s, 2H, -NH of pyrimidine), 8.45 (s, $1 \mathrm{H},-\mathrm{Ar}-\mathrm{H})$; MS: $\mathrm{m} / \mathrm{z}$ 416. Anal. found: $\mathrm{C}, 54.46 ; \mathrm{H}, 3.41 ; \mathrm{F}, 27.31 ; \mathrm{N}, 6.70$. $\mathrm{C}_{19} \mathrm{H}_{14} \mathrm{~F}_{6} \mathrm{~N}_{2} \mathrm{O}_{2}$ requires: $\mathrm{C}, 54.81 ; \mathrm{H}, 3.39 ; \mathrm{F}, 27.38 ; \mathrm{N}$, $6.73 \%$.

\section{6-(3,5-bis(trifluoromethyl)phenyl)-4-(4- (trifluoromethyl)phenyl)-3,4-dihydropyrimidin- 2(1H)-one (4i)}

M.P. ${ }^{66} 6^{\circ} \mathrm{C}$; Yield: $64 \%$; $\mathrm{IR}(\mathrm{KBr})\left(\mathrm{cm}^{-1}\right): 3057(\mathrm{~N}-\mathrm{H})$, $2949(\mathrm{C}-\mathrm{H}), 1623(\mathrm{C}=\mathrm{O})$, 1567, 1446 (C=C), 1025 (CF); 733 (C-Cl); ${ }^{1} \mathrm{H}$ NMR (DMSO- $d 6$ ) $\delta$ ppm: $\delta 5.42$ (s, $2 \mathrm{H}$, - $\mathrm{CH}$ - of pyrimidine ring), $6.72-6.74(\mathrm{~m}, 1 \mathrm{H}, \mathrm{Ar}-$ H), 7.25-7.28 (m, 2H, Ar-H), 7.30-7.35 (s, 1H, -Ar$\mathrm{H}), 7.47-7.50$ (s, 2H, -Ar-H), 8.36-8.39 (s, 2H, -NH of pyrimidine), 8.77 (s, $1 \mathrm{H},-\mathrm{Ar}-\mathrm{H}$ ); MS: $\mathrm{m} / z$ 454. Anal. found: $\mathrm{C}, \quad 50.20 ; \mathrm{H}, 2.40 ; \mathrm{F}, 37.47 ; \mathrm{N}, 6.56$. $\mathrm{C}_{19} \mathrm{H}_{11} \mathrm{~F}_{9} \mathrm{~N}_{2} \mathrm{O}$ requires: $\mathrm{C}, 50.23 ; \mathrm{H}, 2.44 ; \mathrm{F}, 37.64 ; \mathrm{N}$, $6.17 \%$.

\section{6-(3,5-bis(trifluoromethyl)phenyl)-4-(4- nitrophenyl)-3,4-dihydropyrimidin-2(1H)-one (4j)}

M.P. $160^{\circ} \mathrm{C}$; Yield: $69 \%$; $\mathrm{IR}(\mathrm{KBr})\left(\mathrm{cm}^{-1}\right): 3102(\mathrm{~N}-\mathrm{H})$, $2945(\mathrm{C}-\mathrm{H}), 1666(\mathrm{C}=\mathrm{O})$, 1580, $1467(\mathrm{C}=\mathrm{C}), 1054(\mathrm{C}-$ F); 744 (C-Cl); ${ }^{1} \mathrm{H}$ NMR (DMSO- $d 6$ ) $\delta$ ppm: $\delta 5.23$ (s, $2 \mathrm{H}$, - $\mathrm{CH}$ - of pyrimidine ring), 6.90-6.93 $(\mathrm{m}, 1 \mathrm{H}$, Ar$\mathrm{H})$, 7.20-7.25 (m, 2H, Ar-H), 7.40-7.43 (s, 1H, -ArH),7.50-7.54 (s, 2H, -Ar-H), 8.30-8.32 (s, 2H, -NH of pyrimidine), 8.76 (s, $1 \mathrm{H},-$ Ar-H); MS: $m / z$ 431. Anal. found: C, 50.18; H, 2.54; F, 26.51; N, 9.71. $\mathrm{C}_{18} \mathrm{H}_{11} \mathrm{~F}_{6} \mathrm{~N}_{3} \mathrm{O}_{3}$ requires: $\mathrm{C}, 50.13 ; \mathrm{H}, 2.57 ; \mathrm{F}, 26.43 ; \mathrm{N}$, 9.74\%.

\section{BIOLOGICAL EVALUATION}

\section{Antimicrobial evaluation}

All of the synthesized compounds $4 a-j$ were tested for their antibacterial and antifungal activity (MIC) in vitro by broth dilution method ${ }^{8-10}$ with two Grampositive bacteria Staphylococcus aureus MTCC-96, Streptococcus pyogenes MTCC 443, two Gramnegative bacteria Escherichia coli MTCC 442, Pseudomonas aeruginosa MTCC 441 and three fungal strains Candida albicans MTCC 227, Aspergillus Niger MTCC 282, Aspergillus clavatus MTCC 1323 taking ampicillin, chloramphenicol, norfloxacin, nystatin, and griseofulvin as standard drugs. The standard strains were procured from the Microbial Type Culture Collection (MTCC) and Gene Bank, Institute of Microbial Technology, Chandigarh, India. The minimal inhibitory concentration (MIC) values for all the newly synthesized compounds, specified as the humble concentration of the compound preventing the observable growth, were determined by using the microdilution broth method according to NCCLS(National Committee for Clinical Laboratory Standards) standards.

Serial dilutions of the test compounds and reference drugs were prepared in Mueller-Hinton agar. Drugs (10 mg) were dissolved in dimethylsulfoxide (DMSO, $1 \mathrm{~mL}$ ). Further progressive dilutions with melted Mueller-Hinton agar were performed to obtain the required concentrations. In primary screening 1000 $\mu \mathrm{g} \mathrm{mL} L^{-1}, 500 \mu \mathrm{g} \mathrm{mL}^{-1}$ and $250 \mu \mathrm{g} \mathrm{mL}^{-1}$ concentrations of the synthesized drugs were taken. The active synthesized drugs found in this primary screening were further tested in the second set of dilution at $125 \mu \mathrm{g} \mathrm{mL}-1,62.5 \mu \mathrm{g} \mathrm{mL}-1,50 \mu \mathrm{g} \mathrm{mL}-1,25 \mu \mathrm{g} \mathrm{mL}-1$, $12.5 \mu \mathrm{g} \mathrm{mL} \mathrm{m}^{-1}$, and $6.250 \mu \mathrm{g} \mathrm{mL^{-1 }}$ concentration against all microorganisms. The tubes were inoculated with $108 \mathrm{CFU} \mathrm{mL} \mathrm{mL}^{-1}$ (colony-forming unit $/ \mathrm{mL}$ ) and 
incubated at $37 \stackrel{\circ}{\circ}$ for $24 \mathrm{~h}$. The MIC was the lowest concentration of the tested compound that yields no visible growth (turbidity) on the plate. To ensure that the solvent did not affect the bacterial growth, a control was performed with the test medium supplemented with DMSO at the same dilutions as used in the experiments and it was observed that DMSO did not affect the microorganisms in the concentrations studied. The results obtained from antimicrobial susceptibility testing are depicted.

Table 1 :- in vitro Antibacterial Screening Results for $(4 \mathrm{a}-\mathrm{j})$

\begin{tabular}{|c|c|c|c|c|}
\hline \multirow[t]{3}{*}{ Code } & \multicolumn{4}{|c|}{ Minimal inhibition concentration $\left(\mu \mathrm{g} \mathrm{mL}^{-1}\right)$} \\
\hline & \multicolumn{2}{|c|}{ Gram-positive } & \multicolumn{2}{|c|}{ Gram-negative } \\
\hline & GPA1 & GPA2 & GNA3 & GNA4 \\
\hline $4 a$ & 500 & 500 & 1000 & 100 \\
\hline $4 \mathrm{~b}$ & 250 & 250 & 250 & 500 \\
\hline $4 \mathrm{c}$ & 1000 & 1000 & 200 & 250 \\
\hline $4 \mathrm{~d}$ & 250 & 1000 & 250 & 250 \\
\hline $4 \mathrm{e}$ & 1000 & 250 & 250 & 250 \\
\hline $4 \mathrm{f}$ & 250 & 250 & 200 & 250 \\
\hline $4 g$ & 1000 & 500 & 500 & 500 \\
\hline $4 \mathrm{~h}$ & 500 & 500 & 250 & 250 \\
\hline $4 \mathrm{i}$ & 500 & 250 & 100 & 1000 \\
\hline $4 \mathrm{j}$ & 500 & 62.5 & 500 & 100 \\
\hline Ampicillin & 250 & 100 & 100 & 100 \\
\hline Chloramphenicol & 50 & 50 & 50 & 50 \\
\hline \multirow[t]{5}{*}{ Norfloxacin } & 10 & 10 & 10 & 10 \\
\hline & Staphylococcus aureus & GPA1 & & \\
\hline & Streptococcus pyogenes & GPA2 & & \\
\hline & Escherichia coli & GNA3 & & \\
\hline & Pseudomonas aeruginosa & GNA4 & & \\
\hline
\end{tabular}

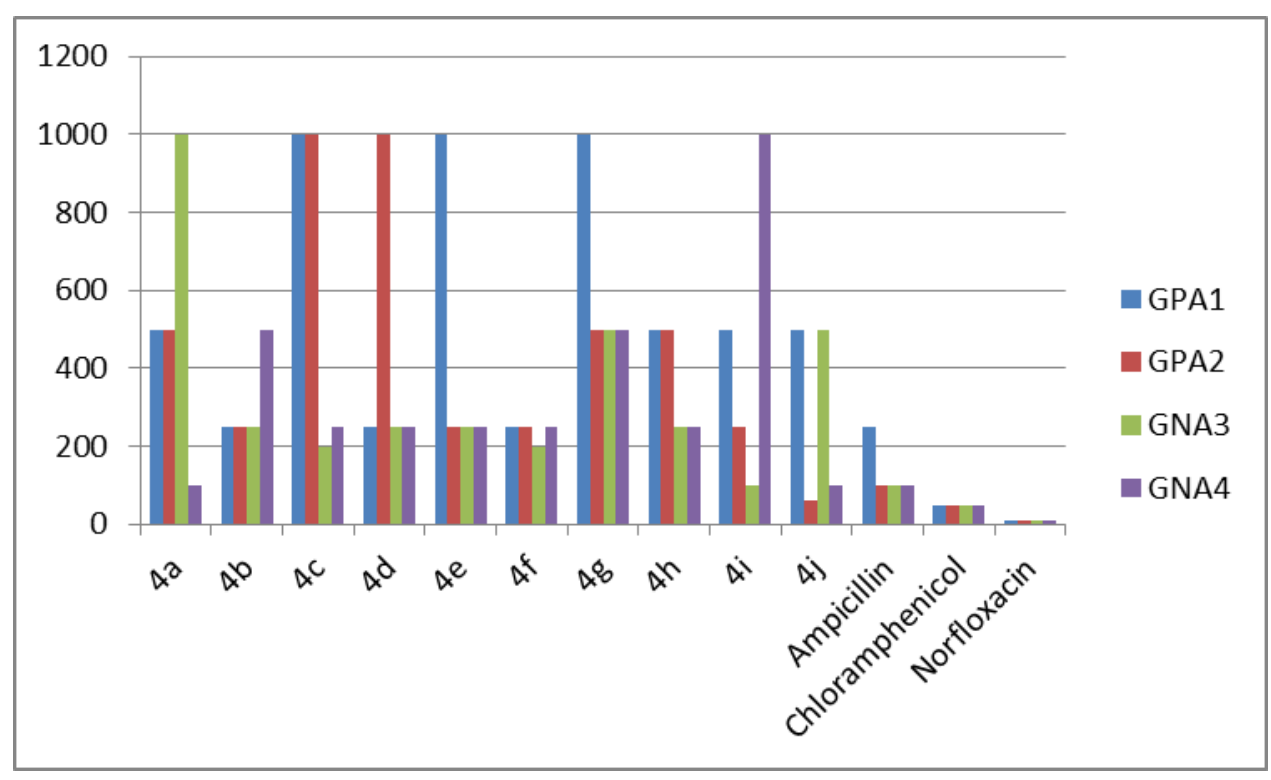


Table 2 :- in vitro Antifungal Screening Results for (4a-j)

\begin{tabular}{|c|c|c|c|}
\hline \multirow[t]{3}{*}{ Code } & \multicolumn{3}{|c|}{ Minimal inhibition concentration $(\mu \mathrm{g}$ mL-1 ) } \\
\hline & \multicolumn{3}{|c|}{ Fungal species } \\
\hline & FSA5 & FSA6 & FSA7 \\
\hline $4 a$ & $>1000$ & 1000 & 500 \\
\hline $4 b$ & 500 & $>1000$ & $>1000$ \\
\hline $4 c$ & $>1000$ & 500 & 250 \\
\hline $4 \mathrm{~d}$ & 500 & $>1000$ & $>1000$ \\
\hline $4 \mathrm{e}$ & $>1000$ & 1000 & 1000 \\
\hline $4 \mathrm{f}$ & 1000 & 500 & 500 \\
\hline $4 g$ & 1000 & $>1000$ & $>1000$ \\
\hline $4 \mathrm{~h}$ & 500 & $>1000$ & $>1000$ \\
\hline $4 \mathrm{i}$ & 500 & 350 & $>1000$ \\
\hline $4 j$ & 350 & 250 & 250 \\
\hline Ampicillin & 400 & 350 & 300 \\
\hline Nystatin & 100 & 100 & 100 \\
\hline Griseofulvin & 450 & 100 & 100 \\
\hline & & Candida albicans & FSA5 \\
\hline & & Aspergillus Niger & FSA6 \\
\hline & & Aspergillus clavatus & FSA7 \\
\hline
\end{tabular}

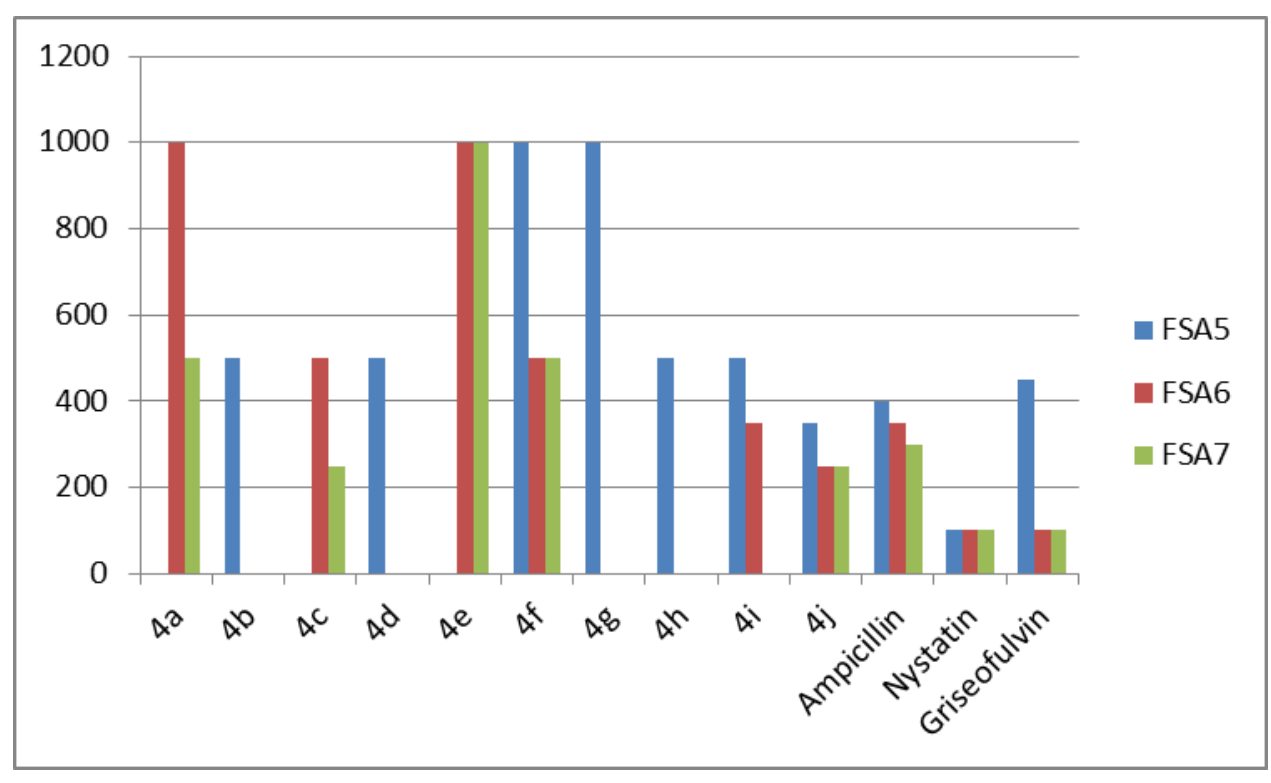

\section{RESULT AND DISCUSSION}

Here we test all synthesized compounds for the determination of their activity for four bacteria in which compound no. $4 \mathrm{~b}$ and $4 \mathrm{f}$ found good inhibitors against staphylococcus aureus and compound no.4b, 4e, 4fand $4 \mathrm{i}$ exhibit good activity at $250 \mu \mathrm{g} \mathrm{mL} \mathrm{m}^{-1}$ against streptococcus pyogenes. 4c, 4i exhibit excellent

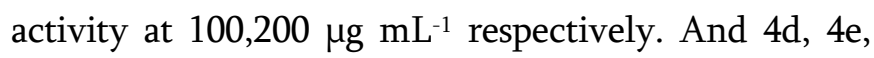
$4 \mathrm{~h}$ are good inhibitors against Escherichia coli. Compound no.4a and $4 \mathrm{j}$ show very good activity 
against pseudomonas aeruginosa while others are moderate.

Three fungal strains are used for screening. In antifungal screening $4 \mathrm{j}$ compound is great inhibitors against all three fungal strains. $4 \mathrm{~b}, 4 \mathrm{~d}, 4 \mathrm{i}$, and $4 \mathrm{j}$ are moderate inhibitors against candida Albicans .4i is an excellent inhibitor while $4 \mathrm{f}$ and $4 \mathrm{c}$ are good inhibitors against the aspergillus Niger .4c exhibit very good antifungal activity at $250 \mu \mathrm{g} \mathrm{mL}^{-1}$ 4f and $4 \mathrm{f}$ are moderate inhibitors.

\section{v. CONCLUSION}

In this paper, we present the method for preparing the triazolopymidine by bignelli reaction and the help of Clausen Schmidt condensation. By comparing results we found many antimicrobial and antifungal agents among them which will use to study of the determination of a variety of activities in triazolopyrimidine

\section{REFERENCES}

[1]. Biginelli, P. (1891). \&quot; Ueber Aldehyduramide des Acetessigäthers\&quot;. Chemische Berichte, 24: 1317-1319. doi:10.1002/cber.189102401228.

[2]. Song, Dailei; Wang, Runxia; Chen, Yongli; Zhang, Shaohua; Liu, Chunsheng; Luo, Genxiang (2008). \&quot;Copper(II) trifluoroacetate catalyzed synthesis of 3,4dihydropyrimidin-2(1H)-ones under solventfree conditions\&quot;. Reaction Kinetics and Catalysis Letters. 95 (2).

[3]. Rovnyak, G. C.; Atwal, K. S.; Hedberg, A.; Kimball, S. D.; Moreland, S.; Gougoutas, J. Z.; O\&\#39;Reilly, B. C.; Schwartz, J.; Malley, M. F. (1992). \& quot; Dihydropyrimidine calcium channel blockers. 4. Basic 3-substituted-4-aryl- 1,4-dihydropyrimidine-5-carboxylic acid esters. Potent antihypertensive agents \& quot;. J. Med. Chem. 35 (17): 3254-3263.

[4]. Reilly, B. C.; Atwal, K. S. (1987). \& quot; Synthesis of Substituted 1,2,3,4-Tetrahydro-6methyl-2-thioxo-5-pyrimidinecarboxylic Acid Esters \& quot Heterocycles. 26 (5): 1189-1192. doi:10.3987/R-1987-05-1189.

[5]. Kumar, Atul; Maurya, Ram Awatar (2007). \&quot;An efficient bakers\&\#39; yeast catalysed synthesis of 3,4-dihydropyrimidin-2-(1H)ones\&quot;. Tetrahedron Letters. 48 (26): 4569.

[6]. Chetan K Jadhav, Amol S Nipate, Asha V Chate, Vishal D Songire, Anil P Patil, Charansingh H Gill (2019) Efficient Rapid Access to Biginelli for the Multicomponent Synthesis of 1,2,3,4-Tetrahydropyrimidines in Room-Temperature Diisopropyl Ethyl Ammonium Acetate, ACS Omega December 31, 4 (27): 22313-22324.

[7]. Shuyan $\mathrm{Yu}$, Jingxin $\mathrm{Wu}$, Hongbing Lan, Lihong Gao, Hengyu Qian, Kaiqi Fan, Zhigang Yin, "palladium and Bronsted acid co-catalyzed biginelli-like multicomponent reactions via in situ generated cyclic enol ether : Access tospirofuran- hydropyrimidines. Organic Letters 2019 December 18.

[8]. National Committee for Clinical and Laboratory Standards, Method for Dilution Antimicrobial Susceptibility Tests for Bacteria that Grow Aerobically Approved Standard, fourth ed. NCCLS, Villanova, Italy, Document M 100-S7, S100-S157 (1997).

[9]. D.H. Isenberg, Essential Procedure for Clinical Microbiology, American Society for Microbiology, Washington, (1998).

[10]. J. R. Zgoda, J. R. Porter, Pharm. Biol., 39, 221225 (2001). 


\section{Cite this article as :}

Jatin Vora, Kartik Vyas, "Synthesis and biological Evaluation of Some Abbreviated New pyrimidine derivatives", International Journal of Scientific Research in Science and Technology (IJSRST), Online ISSN : 2395-602X, Print ISSN : 2395-6011, Volume 6 Issue 3, pp. 354-361, May-June 2019. Available at doi : https://doi.org/10.32628/IJSRST1207250

Journal URL : http://ijsrst.com/IJSRST1207250 